\title{
Polynomial Approximation of a Function and Its First Derivative in Near Minimax Norms*
}

\author{
By Edgar A. Cohen, Jr.
}

\begin{abstract}
Two near minimax norms for polynomial approximation are presented. They are designed for approximation of both a function and its first derivative uniformly by polynomials over a given finite interval. The first one is a convex combination of two integrals, one involving the function and the other the derivative, and the second is the sum of the square of the value of the function at one point and an integral involving the derivative. For any smooth function defined on a finite closed interval, one forms a generalized Chebyshev polynomial expansion to approximate both the function and derivative uniformly.
\end{abstract}

1. Introduction. In any physical problem where it is possible to obtain both position and velocity data, one may want to approximate both sets of data by polynomials simultaneously. If it is desired to approximate the values uniformly, then one could modify the Chebyshev polynomial expansion [4, p. 170] in such a way as to take both of these features into account. In this paper, two norms will be presented, both of which are useful in this respect. In the first procedure, a convex combination of two integrals, one involving the function and the other involving the derivative, is considered. In the second, the square of the value of the function at one point is added to an integral involving the derivative. Thus, in the first case, neither the function nor the derivative data are matched, while, in the second case, the fit is made to coincide with the function data at one arbitrary point of the interval. A simple example is given to illustrate the advantage of using the generalized Chebyshev polynomial expansions, with or without the constraint that the fit match the data at one point, over that of using a standard Chebyshev fit. One immediate practical advantage of either of these procedures over that of Moursund [7] for simultaneous approximation is the guarantee of uniqueness of the approximant. In the work of Meir and Sharma [6], constraints must be imposed to guarantee uniqueness. In addition, there are distinct computational advantages in obtaining best approximants when one employs either of these two methods, since we have at our command all of the knowledge of the structure of inner product spaces [4, pp. 158-197].

\section{Basic Analysis. Let us first consider the norm}

Received January 29, 1970, revised August 18, 1972.

AMS (MOS) subject classifications (1970). Primary 42A60; Secondary 33A65.

Key words and phrases. Function and first derivative, near minimax norms, polynomial approximation, orthogonal basis of polynomials, generalized Chebyshev polynomial expansions, position and velocity data, linear transformation, almost orthogonal set, recurrence relation, normalizing coefficient, best fit, integrals of Chebyshev polynomials, computer programs, Simpson rule, Fourier coefficients, function and derivative deviations, constraint built into norm, derivative deviations near endpoints, convex combination of two integrals, uniqueness of the approximant, error bounds and topological properties.

* Supported by the Naval Ordance Laboratory Independent Research Fund. 


$$
\begin{aligned}
\|g\|^{2}= & \gamma \int_{a}^{b}[(b-y)(y-a)]^{-1 / 2}[g(y)]^{2} d y \\
& +(1-\gamma) \int_{a}^{b}[(b-y)(y-a)]^{-1 / 2}\left[g^{\prime}(y)\right]^{2} d y,
\end{aligned}
$$

where $0<\gamma \leqq 1$ is given. After making the linear transformation

$$
y=\frac{1}{2}[(b-a) x+(b+a)]
$$

and setting $f(x)=g(y)$, we find that

$$
\|g\|^{2}=\|f\|^{2}=\gamma \int_{-1}^{1}\left(1-x^{2}\right)^{-1 / 2}[f(x)]^{2} d x+\delta \int_{-1}^{1}\left(1-x^{2}\right)^{-1 / 2}\left[f^{\prime}(x)\right]^{2} d x,
$$

where $\delta=4(1-\gamma) /(b-a)^{2}$. By dividing (3) by $(\gamma+\delta)$, we transform the problem of finding the best fit to $g$ in (1) by polynomials of degree at most $n$ into one of finding the best fit to $f$ by polynomials of degree at most $n$ in the normalized form

$$
\|f\|^{2}=\beta \int_{-1}^{1}\left(1-x^{2}\right)^{-1 / 2}[f(x)]^{2} d x+(1-\beta) \int_{-1}^{1}\left(1-x^{2}\right)^{-1 / 2}\left[f^{\prime}(x)\right]^{2} d x,
$$

where $0<\beta \leqq 1$ is given. Once we obtain the best fit over $[-1,1]$ in (4), we can use the inverse transformation

$$
x=(2 y-b-a) /(b-a)
$$

to obtain the best fit to $g(y)$. To find the best fit to $f$ in the norm (4), we develop the orthogonal basis of polynomials in (4). If we form the antiderivative $p_{n}(x)$ of the Chebyshev polynomial $T_{n-1}(x)=\cos \left[(n-1) \cos ^{-1} x\right]$, then we have [4, p. 76]

$$
p_{n}(x)=\left[T_{n}(x) / n-T_{n-2}(x) /(n-2)\right] / 2, \quad n \geqq 3 .
$$

In addition, letting

$$
\begin{aligned}
& p_{0}(x)=T_{0}(x)=1, \\
& p_{1}(x)=T_{1}(x) / 2=x / 2, \\
& p_{2}(x)=T_{2}(x) / 4=\left(2 x^{2}-1\right) / 4,
\end{aligned}
$$

we find that the $p_{n}$ 's form an almost orthogonal set, i.e.,

$$
\begin{aligned}
\left(p_{n}, p_{m}\right) \equiv & \beta \int_{-1}^{1}\left(1-x^{2}\right)^{-1 / 2} p_{n}(x) p_{m}(x) d x \\
& +(1-\beta) \int_{-1}^{1}\left(1-x^{2}\right)^{-1 / 2} p_{n}^{\prime}(x) p_{m}^{\prime}(x) d x=0
\end{aligned}
$$

unless $n=m$ or $|n-m|=2$. These polynomials will be used to derive an orthogonal basis of polynomials. To do this, let

$$
\begin{aligned}
& P_{0}(x)=1, \\
& P_{1}(x)=x / 2, \\
& P_{n}(x)=p_{n}(x)+\alpha_{n} P_{n-2}(x), \quad n \geqq 2 .
\end{aligned}
$$


Since, for $n \geqq 4, P_{n}(x)$ must be orthogonal to $p_{n-2}(x)$ and the set $\left\{p_{n}\right\}$ is an almost orthogonal set, we are led to a recurrence relation for the $\alpha_{n}$ 's, namely,

$$
\alpha_{n}=-\left(p_{n}, p_{n-2}\right) /\left[\left(p_{n-2}, p_{n-2}\right)+\alpha_{n-2}\left(p_{n-2}, p_{n-4}\right)\right], \quad n \geqq 4 .
$$

To compute $\alpha_{n}$, we need formulas for $\left(p_{n}, p_{n-2}\right)$ and $\left(p_{n}, p_{n}\right)$. It can be shown directly that

$$
\left(p_{2}, p_{2}\right)=\pi(16-15 \beta) / 32 .
$$

From (6) and the fact that $p_{n}^{\prime}(x)=T_{n-1}(x)$, one sees that

$$
\left(p_{n}, p_{n-2}\right)=-\pi \beta / 8(n-2)^{2}, \quad n \geqq 3,
$$

and that

$$
\left(p_{n}, p_{n}\right)=\pi\left\{(1-\beta)+\beta\left[1 / 4 n^{2}+1 / 4(n-2)^{2}\right]\right\} / 2 .
$$

One also finds that $\alpha_{2}=0, \alpha_{3}=\beta /(2-\beta)$, and $\alpha_{4}=\beta /(16-15 \beta)$. Using (6), (7), (8), and (9), one obtains, for example, when $\beta=\frac{1}{2}$,

$$
\begin{aligned}
& P_{0}(x)=1 \\
& P_{1}(x)=x / 2 \\
& P_{2}(x)=\left(2 x^{2}-1\right) / 4 \\
& P_{3}(x)=\left(4 x^{3}-5 x\right) / 6 \\
& P_{4}(x)=\left(136 x^{4}-200 x^{2}+49\right) / 136 \\
& P_{5}(x)=\left(2064 x^{5}-3420 x^{3}+1265 x\right) / 1290 \\
& P_{6}(x)=\left(37408 x^{6}-69936 x^{4}+34770 x^{2}-2849\right) / 14028
\end{aligned}
$$

Consider the generalized Chebyshev polynomial expansion given by

$$
\sum\left(f, P_{i}\right) P_{i}(x) /\left(P_{i}, P_{i}\right),
$$

where

$$
\left(f, P_{i}\right)=\beta \int_{-1}^{1}\left(1-x^{2}\right)^{-1 / 2} f P_{i} d x+(1-\beta) \int_{-1}^{1}\left(1-x^{2}\right)^{-1 / 2} f^{\prime} P_{i}^{\prime} d x
$$

and

$$
\delta_{i}=\left(P_{i}, P_{i}\right)^{-1 / 2}
$$

is seen to be the normalizing coefficient for $P_{i}$. Then one finds that

$$
\begin{aligned}
& \delta_{0}^{2}=\left(P_{0}, P_{0}\right)^{-1}, \\
& \delta_{1}^{2}=\left(P_{1}, P_{1}\right)^{-1}, \\
& \delta_{n}^{2}=\left[\left(p_{n}, p_{n}\right)+\alpha_{n}\left(p_{n}, p_{n-2}\right)\right]^{-1}, \quad n \geqq 2 .
\end{aligned}
$$

Using (5), the series (12) can now be transformed to a generalized Chebyshev expansion over $[a, b]$, and the first $n+1$ terms of this series give the best polynomial approximation of degree at most $n$ to $g(y)$ in (1). We summarize our results in the form of an algorithm: 
Algorithm 1. To find the best approximating polynomial of a given degree $n$ in the norm (1): $g(y)$.

1. Transform the interval $[a, b]$ by means of $(2)$ to the interval $[-1,1]$ and set $f(x)=$

2. Find an orthogonal basis of polynomials for (4) as follows: Using the integrals of the Chebyshev polynomials as given by (6), together with the relations (7), generate an orthogonal basis of polynomials by use of (8), together with (9) and the fact that $\alpha_{2}=0$, $\alpha_{3}=\beta /(2-\beta)$.

3. Form the generalized Chebyshev polynomial expansion (12), using the relations (13), (14), and (15).

4. Use the inverse transformation (5) to procure the best approximation in the sense of norm (1).

The second norm we shall want to consider is

$$
\|g\|^{2}=[g(c)]^{2}+\int_{a}^{b}[(b-y)(y-a)]^{-1 / 2}\left[g^{\prime}(y)\right]^{2} d y,
$$

where $c \in[a, b]$. However, let us first consider the case where $c=a$. Again, we make the linear transformation (2) and set $f(x)=g(y)$. Then we approximate $f(x)$ by the best polynomial $s_{n}(x)$ of degree at most $n$ in the norm

$$
\|f\|^{2}=[f(-1)]^{2}+\int_{-1}^{1}\left(1-x^{2}\right)^{-1 / 2}\left[f^{\prime}(x)\right]^{2} d x .
$$

After applying (5) and defining

$$
t_{n}(y)=s_{n}(x),
$$

we see that the relation

$$
\left\|f-s_{n}\right\|^{2}=\left[f(-1)-s_{n}(-1)\right]^{2}+\int_{-1}^{1}\left(1-x^{2}\right)^{-1 / 2}\left(f^{\prime}-s_{n}^{\prime}\right)^{2} d x
$$

becomes

$$
\left\|g-t_{n}\right\|^{2}=\left[g(a)-t_{n}(a)\right]^{2}+\frac{1}{4}(b-a)^{2} \int_{a}^{b}[(b-y)(y-a)]^{-1 / 2}\left(g^{\prime}-t_{n}^{\prime}\right)^{2} d y .
$$

Now, since $s_{n}(x)$ is the best fit to $f(x), s_{n}(-1)=f(-1)$, and so $g(a)=t_{n}(a)$. Furthermore, one sees that $t_{n}(y)$ is the best fit to $g(y)$ in the norm

$$
\|g\|^{2}=g^{2}(a)+\frac{1}{4}(b-a)^{2} \int_{a}^{b}[(b-a)(y-a)]^{-1 / 2}\left[g^{\prime}(y)\right]^{2} d y .
$$

Note that the best fit in (18) is the same regardless of the constant appearing before the integral. To find the best fit of given maximal degree $n$, one simply forms the Chebyshev polynomial expansion of degree $n-1$ to the derivative of the function, finds the indefinite integral of the expansion, and adjusts the constant term so that $t_{n}(a)=g(a)$. To do this, one may proceed as follows: Let

$$
\begin{aligned}
q_{k}(x) & =\int_{-1}^{x} T_{k-1}(t) d t=\left(x^{2}-1\right) / 2, \quad k=2, \\
& =\frac{1}{2}\left[T_{k}(x) / k-T_{k-2}(x) /(k-2)+2(-1)^{k} / k(k-2)\right], \quad k \geqq 3 .
\end{aligned}
$$


Then it is seen $[4, p .170]$ that

$$
s_{n}(x)=f(-1)+\frac{1}{2} a_{1}(x+1)+\sum_{k=2}^{n} a_{k} q_{k}(x),
$$

where

$$
a_{k} \equiv 2 \int_{-1}^{1}\left(1-x^{2}\right)^{-1 / 2} f^{\prime} T_{k-1} d x / \pi
$$

We again summarize our results in the form of an algorithm:

Algorithm 2. To find the best approximating polynomial of degree $n$ in the norm (18):

1. Transform the interval $[a, b]$ by means of $(2)$ to the interval $[-1,1]$ and set $f(x)=$ $g(y)$.

2. Form the polynomial expansion (20), using the indefinite integral (19) and the Fourier coefficients $a_{k}$ as given by (21).

3. Use the inverse transformation (5) to procure the best approximation in the sense of (18).

To obtain the best $t_{n}$ subject to the condition $t_{n}(c)=g(c)$, one now adds to the $t_{n}$ obtained for (18) the deviation $g(c)-t_{n}(c)$. By so altering the constant term in $t_{n}$, one gets the best approximant of degree $n$ in (16).

3. Error Bounds and Topological Properties. In order to obtain an error estimate for the norm (4), we shall first of all examine in more detail the structure of the sequence $\left\{\alpha_{n}\right\}$ as given recursively in (9). A number of properties can be established by mathematical induction. First of all, from (9), (10), and (11), together with the expressions for $\alpha_{2}, \alpha_{3}$, and $\alpha_{4}$, it follows easily by induction that $\left|\alpha_{n}\right|<1$ for all $n$ whenever $|\beta|<1$. It also follows by induction that $\alpha_{n}$ is analytic in the disk $|\beta|<1$, has a simple zero at $\beta=0$, and is strictly increasing on $0 \leqq \beta \leqq 1$ from 0 at $\beta=0$ to 1 at $\beta=1$. It is clear, in addition, that $\alpha_{n}$ is a rational function of $\beta$ for every $n$. From the fact now that $\left|\alpha_{n}\right|<1$ whenever $|\beta|<1$ and from (9), (10), and (11), it follows that, for $|\beta|<1, \alpha_{n}$ is asymptotic to $\beta / 4(1-\beta) n^{2}$ as $n \rightarrow \infty$. It can thus be established that there exists a positive constant $K(\beta)$ such that, whenever $n \geqq 3$ and $0 \leqq \beta<1$,

$$
\alpha_{n} \leq \beta K(\beta) / 4(1-\beta) n^{2} .
$$

With this information, we can secure, in the first place, an upper bound for $\left\{P_{n}(x)\right\}$. From (6), (7), (8), and (22), one finds that, for $n \geqq 3$ and $0 \leqq \beta<1$,

$$
\begin{aligned}
\left|P_{n}(x)\right| & \leqq \frac{4}{3}\left[\sum_{k=1}^{(n / 2]}\left(\prod_{i=1}^{k} \alpha_{n-2 i+2}\right)\right]+\frac{1}{2}(1 / n+1 /(n-2)) \\
& <\frac{4}{3}([n / 2]) \alpha_{n}+\frac{1}{2}(1 / n+1 /(n-2)) \\
& \leqq \beta K(\beta) / 6 n(1-\beta)+\frac{1}{2}(1 / n+1 /(n-2)) \\
& \leqq c_{\beta} / n,
\end{aligned}
$$

where, for a given $\beta<1, c_{\beta}$ is a constant. Also, from (6) and (8), together with the result above, it can be seen that 


$$
\left|P_{n}(x)\right| \leqq \frac{1}{2}(1 / n+1 /(n-2))+d_{\beta} / n^{3} \leqq \kappa / n,
$$

where $d_{\beta}$ is another constant which is dependent upon $\beta$. Note that, when $\beta=0$, the series (12) becomes

$$
\sum\left(f, p_{i}\right) p_{i}(x) /\left(p_{i}, p_{i}\right)=2 \sum\left(f, p_{i}\right) p_{i} / \pi,
$$

where

$$
\left(f, p_{i}\right)=\int_{-1}^{1} f^{\prime}(x) T_{i-1}(x)\left(1-x^{2}\right)^{-1 / 2} d x
$$

This series does not seem to interpolate to $f$ at any preassigned point of $[-1,1]$. In contrast, see [3]. To obtain an error estimate for (12) when $n$ terms are used, we shall first investigate the leading term in the remainder series, namely, $\left(f, P_{n}\right) P_{n}(x) /\left(P_{n}, P_{n}\right)$.

We already have a bound for $P_{n}(x)$. Now we must determine the behavior of $\left(f, P_{n}\right)$ and $\left(P_{n}, P_{n}\right)$. Suppose that $f^{\prime}$ is continuous and of bounded variation on $[-1,1]$. Then it is well known [2] that

$$
A_{n} \equiv \int_{-1}^{1} f(x) T_{n}(x)\left(1-x^{2}\right)^{-1 / 2} d x
$$

is $O\left(1 / n^{2}\right)$ and $[9, \mathrm{p} .24]$

$$
B_{n} \equiv \int_{-1}^{1} f^{\prime}(x) T_{n}(x)\left(1-x^{2}\right)^{-1 / 2} d x
$$

is $O(1 / n)$ as $n \rightarrow \infty$. Now

$$
\left(f, P_{n}\right)=\left(f, p_{n}\right)+\alpha_{n}\left(f, P_{n-2}\right),
$$

and it is clear that $\alpha_{n}\left(f, P_{n-2}\right)=O\left(1 / n^{2}\right)$. Also,

$$
\left(f, p_{n}\right)=\beta\left[A_{n} / n-A_{n-2} /(n-2)\right] / 2+(1-\beta) B_{n-1} .
$$

From the almost orthogonal property of the set $\left\{p_{n}\right\}$, together with (10) and (11), one sees that

$$
\begin{aligned}
\left(P_{n}, P_{n}\right) & =\left(p_{n}, p_{n}\right)+\alpha_{n}\left(p_{n}, p_{n-2}\right) \\
& =\pi\left[(1-\beta)+\beta\left(1 / 4 n^{2}+1 / 4(n-2)^{2}\right)\right] / 2-\pi \beta \alpha_{n} / 8(n-2)^{2} .
\end{aligned}
$$

Under our hypotheses on $f$ and $f^{\prime}$, the bracketed term in (26) is $O\left(1 / n^{3}\right)$, and the remaining term is $O(1 / n)$. If, in both (25) and (27), we can neglect terms which are definitely $O\left(1 / n^{2}\right)$, we find that $a_{n} \equiv\left(f, P_{n}\right) /\left(P_{n}, P_{n}\right)$ is given approximately by $2 B_{n-1} / \pi$, independent of $\beta$, for $0 \leqq \beta \leqq 1-\epsilon$, where $\epsilon>0$ is chosen a priori. In any event, using a line of reasoning suggested by Blum and Curtis [1], let us assume that, for a given $\beta,\left|a_{k+1}\right| \leqq \rho\left|a_{k}\right|$, where $k \geqq n$ and $\rho<1$. Then, letting $E_{n}$ represent the error between $f(x)$ and the first $n$ terms of the generalized Chebyshev polynomial expansion, we have

$$
\begin{aligned}
E_{n} & \leqq \sum_{k=n}^{\infty}\left|a_{k}\right|\left|P_{k}(x)\right| \leqq \kappa \sum_{n}^{\infty}\left|a_{k}\right| / k \leqq \kappa \sum_{n}^{\infty}\left|a_{k}\right| / n \\
& \leqq \kappa\left|a_{n}\right| / n(1-\rho) \simeq\left|B_{n-1}\right|(1 / n+1 /(n-2)) /(1-\rho) \pi,
\end{aligned}
$$


as can be seen from (23) and the above result, provided we also neglect the term of order $1 / n^{3}$ in (23). Since $P_{n}^{\prime}(x)$ is $O(1)$, we can, arguing in the same way, say that the error $E_{n}^{\prime}$ between $f^{\prime}(x)$ and the derived series truncated after $n$ terms is bounded above roughly by $2\left|B_{n-1}\right| / \pi(1-\rho)$. It is clear that, as $\beta \rightarrow 1$, the approximation error, for a given $n$, will become worse, since the generalized Chebyshev expansion then approaches the classical Chebyshev expansion. However, if one first picks $\epsilon>0$, then, within the interval $0 \leqq \beta \leqq 1-\epsilon, E_{n}$ and $E_{n}^{\prime}$ are given essentially as indicated above, provided $n$ is chosen sufficiently large. When one differentiates the classical Chebyshev expansion, he obtains an expansion in Chebyshev polynomials of the second kind [5], which may not even converge to $f^{\prime}(x)$ at the endpoints of the interval $[-1,1]$. Bounds for the error upon using the norm (16) are of the same order of accuracy as for the norm (1). They can, in fact, be obtained easily from the work of Clenshaw and Curtis [2]. The reason for use of the norm (16) would be that one had a problem for which it was required that the function be duplicated at a specified point of the interval $[a, b]$.

There is an interesting topological connection between the norm (1) and the norm (16). We shall, without loss of generality, assume that $a=-1, b=1$. Also, we assume in what follows that $g(x)$ is absolutely continuous and has a square-summable derivative $[8, \mathrm{pp} .165,243]$. Let

$$
M=\int_{-1}^{1} g^{2}(x)\left(1-x^{2}\right)^{-1 / 2} d x \quad \text { and } \quad M^{\prime}=\int_{-1}^{1} g^{\prime}(x)^{2}\left(1-x^{2}\right)^{-1 / 2} d x .
$$

It is easy to see, first of all, that there exists at least one $x$, say $x_{0}$, in $(-1,1)$ such that

$$
g^{2}\left(x_{0}\right) \leqq \frac{1}{2} \int_{-1}^{1} g^{2}(x)\left(1-x^{2}\right)^{-1 / 2} d x=M / 2
$$

For, if not, then, for every $x$ in $(-1,1)$,

$$
g^{2}(x)\left(1-x^{2}\right)^{-1 / 2} \geqq g^{2}(x)>M / 2
$$

from which, upon integrating both sides between -1 and 1 , we have an immediate contradiction. Now

$$
g\left(x_{0}\right)-g(c)=\int_{c}^{x_{0}} g^{\prime}(t) d t=\int_{c}^{x_{0}}\left(1-t^{2}\right)^{1 / 4}\left(1-t^{2}\right)^{-1 / 4} g^{\prime}(t) d t .
$$

By the Schwarz inequality [4, p. 134], it follows that $\left[g\left(x_{0}\right)-g(c)\right]^{2} \leqq \pi M^{\prime} / 2$. So

$$
|g(c)| \leqq\left|g\left(x_{0}\right)\right|+\left|g\left(x_{0}\right)-g(c)\right| \leqq(M / 2)^{1 / 2}+\left(\pi M^{\prime} / 2\right)^{1 / 2},
$$

and, therefore, upon using the elementary inequality

$$
(a+b)^{2} \leqq 2 a^{2}+2 b^{2},
$$

we find

$$
|g(c)|^{2} \leqq M+\pi M^{\prime}
$$

Thus

$$
|g(c)|^{2}+M^{\prime} \leqq \beta M / \beta+(\pi+1)(1-\beta) M^{\prime} /(1-\beta) .
$$

If then $k_{\beta}=\max [1 / \beta,(\pi+1) /(1-\beta)]$, we have 


$$
|g(c)|^{2}+M^{\prime} \leqq k_{\beta}\left(\beta M+(1-\beta) M^{\prime}\right) .
$$

Also,

$$
|g(x)| \leqq|g(c)|+\left|\int_{c}^{x} g^{\prime}(t) d t\right| \leqq|g(c)|+\left(\pi M^{\prime} / 2\right)^{1 / 2}
$$

upon applying the Schwarz inequality a second time. Thus, using (28) again,

$$
\begin{aligned}
M & \leqq \int_{-1}^{1}\left[|g(c)|+\left(\pi M^{\prime} / 2\right)^{1 / 2}\right]^{2}\left(1-t^{2}\right)^{-1 / 2} d t \\
& \leqq 2 \pi\left[|g(c)|^{2}+\pi M^{\prime} / 2\right] .
\end{aligned}
$$

So

$$
\beta M+(1-\beta) M^{\prime} \leqq 2 \pi \beta|g(c)|^{2}+\pi^{2} \beta M^{\prime}+(1-\beta) M^{\prime} .
$$

Letting $m_{\beta}=\max \left[2 \pi \beta,\left(\pi^{2}-1\right) \beta+1\right]$, we have

$$
\beta M+(1-\beta) M^{\prime} \leqq m_{\beta}\left(|g(c)|^{2}+M^{\prime}\right) .
$$

Incorporating (29) and (30) into one inequality, we have, for $0<\beta<1$,

$$
|g(c)|^{2}+M^{\prime} \leqq k_{\beta}\left(\beta M+(1-\beta) M^{\prime}\right) \leqq k_{\beta} m_{\beta}\left(|g(c)|^{2}+M^{\prime}\right) .
$$

This establishes a direct topological connection between the two norms.

4. Computer Calculations. Two computer programs to find best fits over a given closed interval $[a, b]$ have been written, one for each norm. In both programs, the fifth derivative of $g(y)$ is assumed continuous, since Simpson's rule is used to numerically evaluate integrals involving both the function and its derivative. The Fourier coefficients in each norm, namely,

$$
b_{i}=\left(f, P_{i}\right) /\left(P_{i}, P_{i}\right), \quad a_{i}=2 \int_{-1}^{1}\left(1-x^{2}\right)^{-1 / 2} f^{\prime} T_{i-1} d x / \pi
$$

are calculated and printed. The Chebyshev series is then transformed to $[a, b]$, the terms grouped, and the coefficients of the powers of $y$ obtained are written. Lastly, the function and derivative deviations are printed.

As a sample case, the function $g(y)=\exp \left(-y^{2}\right)$ was considered. The following graphs show the deviations $g(y)-t_{n}(y)$ and $g^{\prime}(y)-t_{n}^{\prime}(y)$ over $[0,1]$. Fig. 1 shows the results when the classical norm

$$
\|g\|^{2}=\int_{0}^{1} g^{2} y^{-1 / 2}(1-y)^{-1 / 2} d y
$$

is used subject to the constraint $t_{4}(0)=g(0)$. In comparison, Fig. 2 shows the deviations when the norm

$$
\|g\|^{2}=g^{2}(0)+\int_{0}^{1}\left(g^{\prime}\right)^{2} y^{-1 / 2}(1-y)^{-1 / 2} d y
$$

is employed. In this case, the constraint is the same, but is built into the norm. In contrast to Fig. 2, one sees in Fig. 1 a large derivative deviation near $y=0$ and $y=1$. 


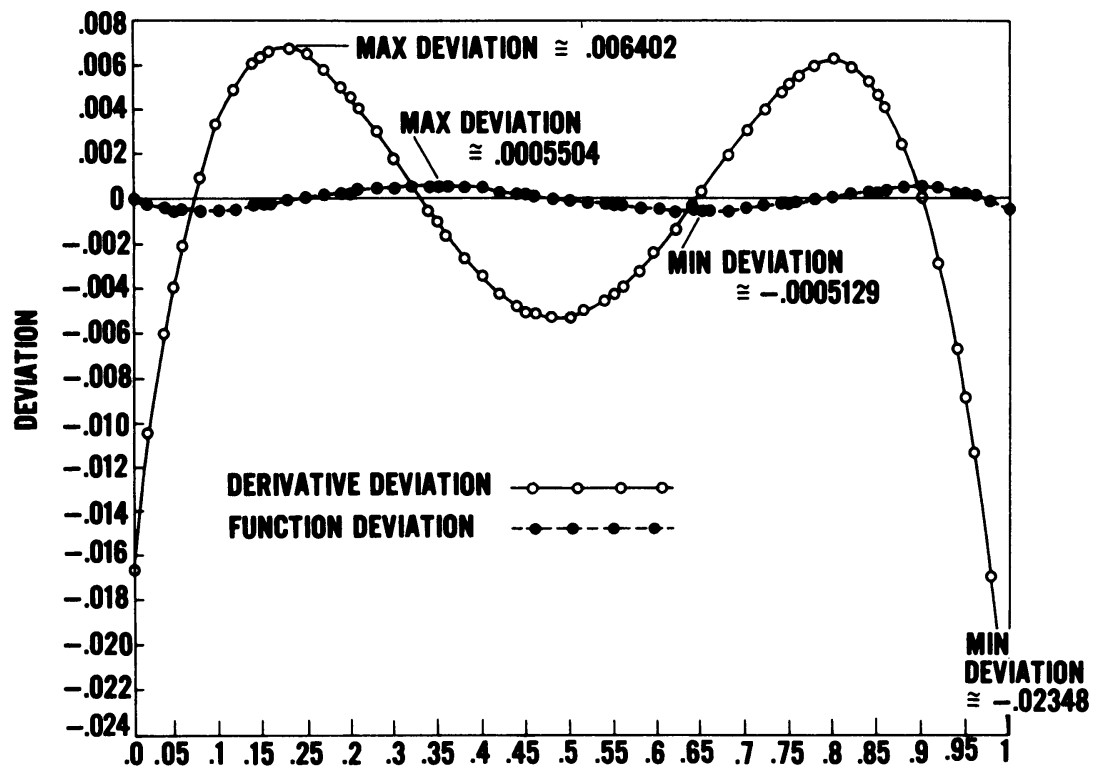

Figure 1. Best Polynomial Fit of Degree 4 to $\exp \left(-y^{2}\right)$ over $[0,1]$ Using $\|g\|^{2}=\int_{0}^{1} g^{2} y^{-1 / 2}(1-y)^{-1 / 2} d y$ and Constraint $g(0)=t_{4}(0)$.

The classical norm (32) gives a smaller deviation away from the ends of the interval, but the overall aspects of the fit to the derivative are better using norm (33), as can be seen in the second figure. Fig. 3 illustrates the case where again the classical norm (32) is used, but the fit is not forced to coincide with the function at $y=0$. Fig. 4 shows the results when the norm

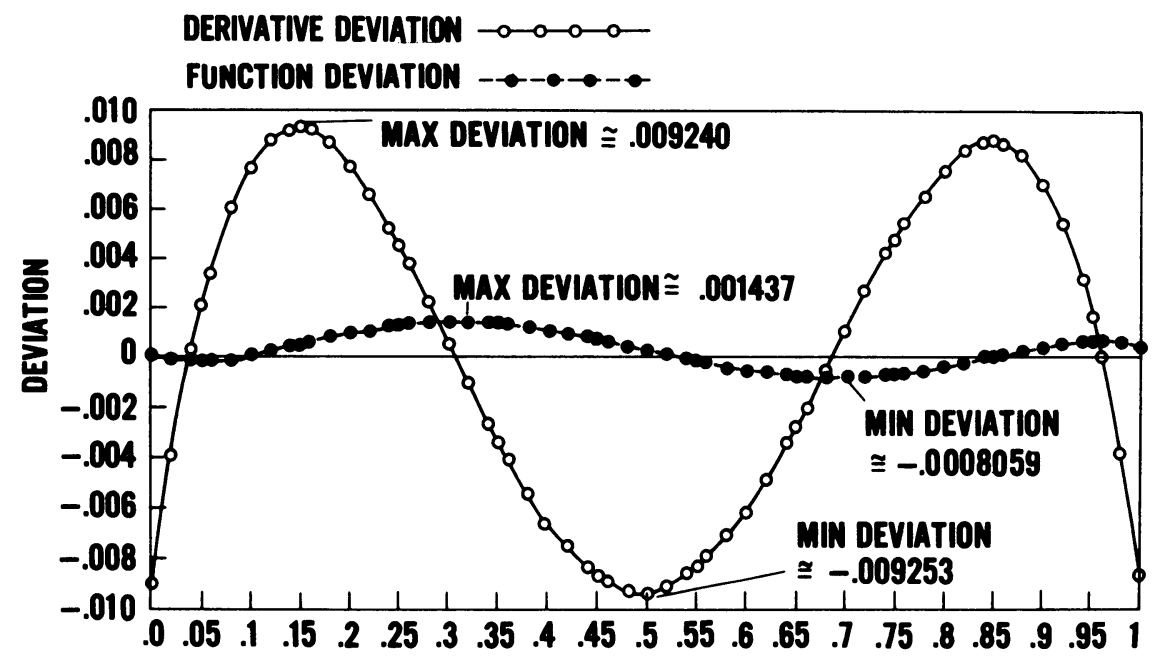

Figure 2. Best Polynomial Fit of Degree 4 to $\exp \left(-y^{2}\right)$ over $[0,1]$ Using

$$
\|g\|^{2}=g^{2}(0)+\int_{0}^{1}\left(g^{\prime}\right)^{2} y^{-1 / 2}(1-y)^{-1 / 2} d y .
$$




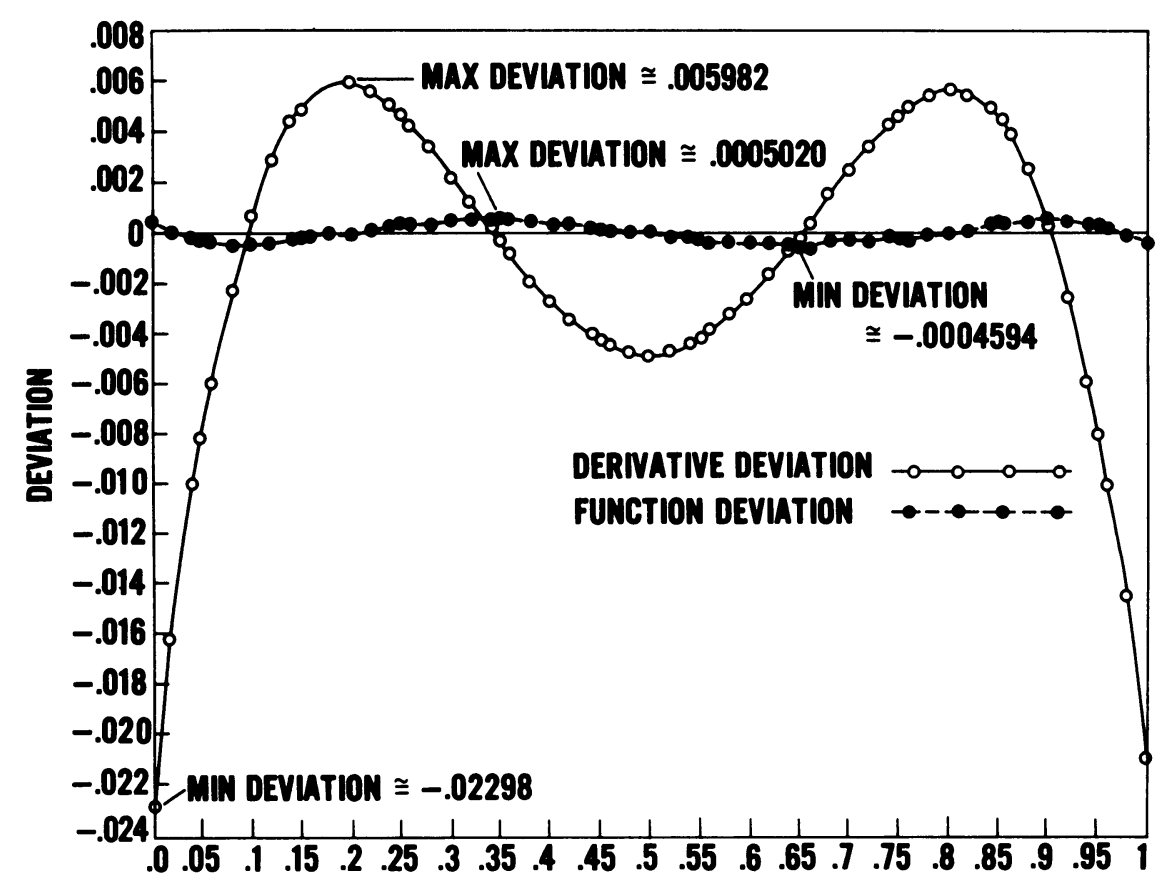

Figure 3. Best Polynomial Fit of Degree 4 to $\exp \left(-y^{2}\right)$ over $[0,1]$ Using $\|g\|^{2}=\int_{0}^{1} g^{2} y^{-1 / 2}(1-y)^{-1 / 2} d y$, with No Constraints.

$$
\|g\|^{2}=\int_{0}^{1} g^{2} y^{-1 / 2}(1-y)^{-1 / 2} d y+\left(\frac{1}{2}\right)^{2} \int_{0}^{1}\left(g^{\prime}\right)^{2} y^{-1 / 2}(1-y)^{-1 / 2} d y
$$

is employed. This norm is used to make both the function and derivative deviations small without restricting the fit to match the function at the origin. Thus it is fair to compare results obtained in these two cases. We see that again the derivative deviations

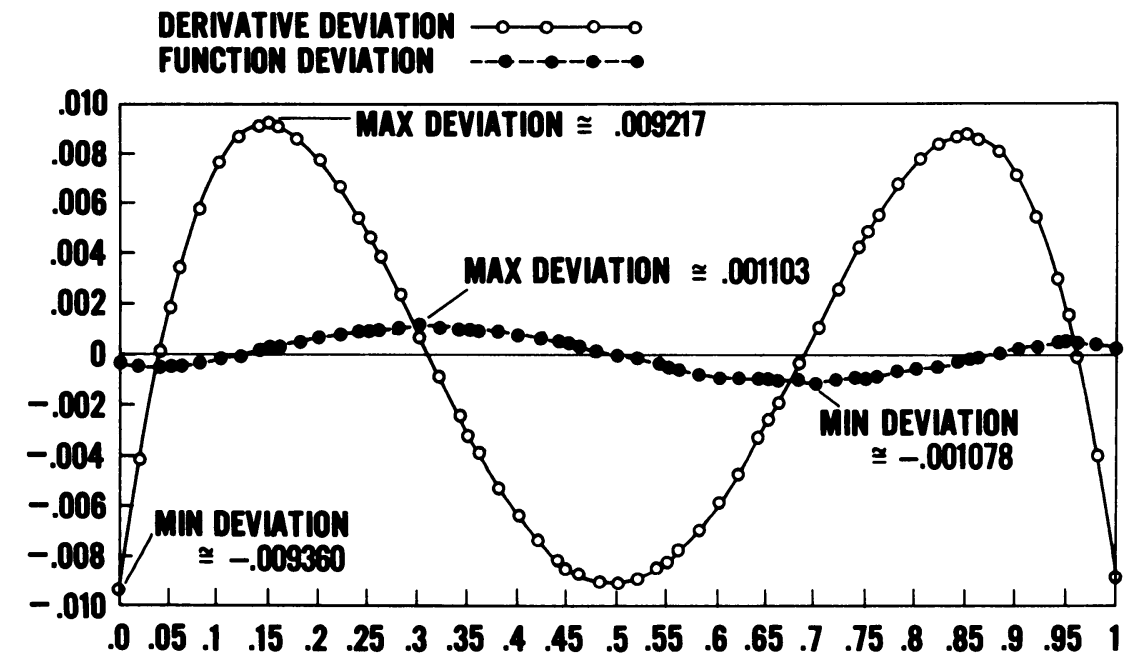

Figure 4. Best Polynomial Fit of Degree 4 to $\exp \left(-y^{2}\right)$ over $[0,1]$ Using $\|g\|^{2}=\int_{0}^{1} g^{2} y^{-1 / 2}(1-y)^{-1 / 2} d y+\left(\frac{1}{2}\right)^{2} \int_{0}^{1}\left(g^{\prime}\right)^{2} y^{-1 / 2}(1-y)^{-1 / 2} d y$. 
for the classical norm are smaller over the central portion, but that, for the norm (34), they are noticeably smaller near the ends. As far as function deviations are concerned, the classical norm gives better results. This is to be expected, since norms (33) and (34) are intended to fit the function and its derivative simultaneously. Notice that there is very little difference between Figs. 2 and 4 except for the matching condition at the left endpoint. This is in agreement with the arguments in Section 3.

Mathematical Analysis Division

Naval Ordnance Laboratory

White Oak

Silver Spring, Maryland 20910

1. E. K. Blum \& P. C. CURTIS, JR., "Asymptotic behavior of the best polynomial approximation," J. Assoc. Comput. Mach., v. 8, 1961, pp. 645-647. MR 24 \#A382.

2. C. W. ClenshaW \& A. R. CURTIS, "A method for numerical integration on an automatic computer," Numer. Math., v. 2, 1960, pp. 197-205. MR 22 \#8659.

3. E. A. COHEN, JR., "Theoretical properties of best polynomial approximation in $W^{1,2}[-1,1]$," SIAM J. Math. Anal., v. 2, 1971, pp. 187-192. MR 45 \#4031.

4. P. J. DAvIs, Interpolation and A pproximation, Blaisdell, New York, 1963. MR 28 \#393.

5. S. FILIPPI, "Angenäherte Tschebyscheff-Approximation einer Stammfunktion-eine Modifikation des Verfahrens von Clenshaw und Curtis," Numer. Math., v. 6, 1964, pp. 320328. MR 30 \#710.

6. A. MiER \& A. Sharma, "Simultaneous approximation of a function and its derivatives," SIAM J. Numer. Anal., v. 3, 1966, pp. 553-563. MR 35 \#7044.

7. D G. Moursund, "Chebyshev approximations of a function and its derivatives," Math. Comp., v. 18, 1964, pp. 382-389. MR 29 \#3804.

8. I. P. NATANSON, Theory of Functions of a Real Variable. Vol. 1, GITTL, Moscow, 1950; English transl., Ungar, New York, 1955. MR 12, 598; MR 16, 804.

9. W. Rogosinski, Fourier Series, Chelsea, New York, 1959. 